\title{
Population Pharmacokinetic Modelling of Recombinant Factor IX Fc Fusion Protein (rFIXFc) in Patients with Haemophilia B
}

\author{
Lei Diao - Shuanglian Li · Thomas Ludden • \\ Jogarao Gobburu • Ivan Nestorov • Haiyan Jiang
}

Published online: 23 January 2014

(c) The Author(s) 2014. This article is published with open access at Springerlink.com

\begin{abstract}
Background and Objectives Recombinant factor IX Fc fusion protein (rFIXFc) is a clotting factor developed using monomeric Fc fusion technology to prolong the circulating half-life of factor IX. The objective of this analysis was to elucidate the pharmacokinetic characteristics of rFIXFc in patients with haemophilia $\mathrm{B}$ and identify covariates that affect rFIXFc disposition.

Methods Population pharmacokinetic analysis using NONMEM $^{\circledR}$ was performed with clinical data from two completed trials in previously treated patients with severe to moderate haemophilia $\mathrm{B}$. Twelve patients from a phase $1 / 2$ a study and 123 patients from a registrational phase 3 study were included in this population analysis.

Results A three-compartment model was found to best describe the pharmacokinetics of rFIXFc. For a typical $73 \mathrm{~kg}$ patient, the clearance (CL), volume of the central compartment $\left(V_{1}\right)$ and volume of distribution at steady
\end{abstract}

L. Diao and S. Li contributed equally to this work.

Electronic supplementary material The online version of this article (doi:10.1007/s40262-013-0129-7) contains supplementary material, which is available to authorized users.

L. Diao $\cdot$ S. Li $\cdot$ I. Nestorov $\cdot$ H. Jiang $(\bowtie)$

Biogen Idec, 14 Cambridge Center,

Cambridge, MA 02142, USA

e-mail: haiyan.jiang@biogenidec.com

T. Ludden

12102 Carson Cove, San Antonio, TX 78253, USA

J. Gobburu

Department of Pharmacy Practice and Science, Center for

Translational Medicine, School of Pharmacy, University of

Maryland, 20 North Pine Street, Baltimore, MD 21201, USA state $\left(V_{\mathrm{ss}}\right)$ were $2.39 \mathrm{dL} / \mathrm{h}, 71.4 \mathrm{dL}$ and $198 \mathrm{dL}$, respectively. Because of repeat pharmacokinetic profiles at week 26 for patients in a subgroup, inclusion of interoccasion variability (IOV) on CL and $V_{1}$ were evaluated and significantly improved the model. The magnitude of IOV on CL and $V_{1}$ were both low to moderate $(<20 \%)$ and less than the corresponding inter-individual variability. Body weight (BW) was found to be the only significant covariate for rFIXFc disposition. However, the impact of BW was limited, as the BW power exponents on CL and $V_{1}$ were 0.436 and 0.396 , respectively.

Conclusion This is the first population pharmacokinetic analysis that systematically characterized the pharmacokinetics of long-lasting rFIXFc in patients with haemophilia B. The population pharmacokinetic model for rFIXFc can be utilized to evaluate and optimize dosing regimens for the treatment of patients with haemophilia B.

\section{Background}

Haemophilia B is a rare bleeding disorder caused by a deficiency of coagulation factor IX (FIX). The disease is caused by mutations in the gene for FIX on the $\mathrm{X}$ chromosome and affects approximately one in 30,000 males [1, 2]. Haemophilia $\mathrm{B}$ results in inadequate clot formation, causing prolonged and abnormal bleeding, including bleeding into joints, soft tissue, muscle and body cavities. Bleeding episodes may be associated with trauma or occur in the absence of trauma (spontaneous bleeding). If not treated appropriately, bleeding can be life threatening or result in significant morbidity [2, 3]. The current mainstay of treatment is FIX replacement therapy. 
Recombinant factor IX Fc fusion protein $(\mathrm{rFIXFc})$ consists of a single molecule of FIX covalently fused to the Fc domain of human immunoglobulin G1 (IgG1) with no intervening sequence. The $\mathrm{Fc}$ domain is responsible for the long circulating half-life of IgG1 through interaction with the neonatal $\mathrm{Fc}$ receptor $(\mathrm{FcRn})$, which is expressed in many different cell types $[4,5]$. rFIXFc was therefore designed to have a prolonged half-life relative to recombinant factor IX (rFIX) [6, 7]. rFIXFc has the potential to fulfil an unmet medical need and decrease treatment burden by providing a long-lasting therapy for control and prevention of bleeding episodes, routine prophylaxis and perioperative management in patients with haemophilia $\mathrm{B}$. Two clinical trials with rFIXFc have been completed in previously treated patients with severe to moderate haemophilia B [with $\leq 2 \mathrm{IU} / \mathrm{dL}(\%)$ endogenous FIX]: one single-ascending-dose phase $1 / 2$ a study in 14 patients (12 of them who received doses $\geq 12.5 \mathrm{IU} / \mathrm{kg}$ had pharmacokinetic assessment) [6], and one registrational phase 3 study in 123 patients [8]. rFIXFc was shown to be well tolerated and efficacious in the treatment of bleeding, routine prophylaxis and perioperative management [8].

The purpose of this analysis was to characterize the population pharmacokinetics of $\mathrm{rFIXFc}$ in patients with haemophilia B and to identify demographic and clinical factors that are potential determinants of rFIXFc pharmacokinetic variability. Additionally, we assessed the ability of the population pharmacokinetic model of rFIXFc to predict FIX activity and thus evaluate and guide dosing regimens of $\mathrm{rFIXFc}$ in the treatment of patients with haemophilia B.

\section{Methods}

\subsection{Clinical Studies}

FIX activity data were obtained from two completed clinical trials in previously treated patients with severe to moderate haemophilia B. Twelve evaluable patients from the phase $1 / 2 \mathrm{a}$ study and 123 patients from the phase 3 study (B-LONG) who had measurable FIX activities were included in this population pharmacokinetic analysis $[6,8]$. The clinical studies are summarized in Fig. 1a, b. The trials were registered at http://www.clinicaltrials.gov as NCT00716716 (phase 1/2a) and NCT01027364 (phase 3). All subjects were patients with severe to moderate haemophilia B previously treated with FIX products, from 12.1 to 76.8 years of age. All patients, or patient guardians, gave written informed consent. The studies were approved by the ethics committee and conducted in accordance with the International Conference on Harmonisation guidelines for Good Clinical Practice.

\subsection{Pharmacokinetic Sampling and Bioanalytical Methods}

In the phase $1 / 2 \mathrm{a}$ study, 12 patients underwent $\mathrm{rFIXFc}$ pharmacokinetic sampling up to 14 days. In the phase 3 study, pharmacokinetic samples were collected for rFIXFc in all patients according to the schedule in Fig. 1c. Pharmacokinetic profiles of $\mathrm{rFIXFc}$ were assessed at week 1 (baseline) for all patients and at week 26 for the Arm 1 sequential pharmacokinetic subgroup. For patients on prophylaxis in Arms 1 and 2, additional trough and peak samples were collected at clinical visits throughout the study.

The population pharmacokinetic modelling was performed using plasma FIX activity data as measured by the one-stage activated partial thromboplastin time (aPTT) clotting assay using commercially available aPTT reagents (Trinity Biotech) and normal reference plasma (Precision BioLogic). The lower limit of quantitation (LLOQ) was $1 \mathrm{IU} / \mathrm{dL}(\%)$. The accuracy of the assay was within 95-104 \%, and the intra- and inter-assay precision was approximately $10 \%$.

\subsection{Data Handling}

A total of 11 data post infusion were below the limit of quantification (BLQ, below LLOQ of $1 \%$ ). Since those post infusion BLQ values represented $<0.5 \%$ of the observations, they were excluded from the analysis as the first step of data handling [9-11].

The one-stage clotting assay does not distinguish between FIX activities resulting from the input study drug, rFIXFc, endogenous baseline FIX or residual activity of the pre-study FIX product due to incomplete washout. Therefore, the baseline and residual activity corrections were applied to the observed FIX activity data (Eqs. 1 and 2). The corrected FIX activities were recorded as the dependent variable (DV) in the population pharmacokinetic dataset. Similar baseline and residual activity corrections were reported previously for the pharmacokinetic analyses of other FIX products [12-15].

Residual decay correction $=($ Pre-dose - baseline $)$

$$
\times \mathrm{e}^{- \text {decayrate } \times \text { time }}
$$

Corrected FIX activity = Measured FIX activity

- baseline - residual decay correction

The endogenous baseline FIX activity level is dictated by the defective FIX genotype and thus is stable in each individual subject, yet could be overestimated in patients receiving FIX replacement therapy who underwent incomplete washout. Therefore, the baseline FIX activity was defined as the lowest FIX activity observed throughout the study, including all the screening, pre-dose and post- 


\section{a Phase 1/2a Study Design}

\begin{tabular}{|c|c|c|c|}
\hline $\begin{array}{c}\text { Screening } \\
(~ 14 \text { days })\end{array}$ & $\begin{array}{c}\text { Washout } \\
(\sim 7 \text { days })\end{array}$ & $\begin{array}{c}\text { Safety \& pharmacokinetics } \\
\text { (3-17 days })\end{array}$ & $\begin{array}{c}\text { Follow-up } \\
\text { (30 days post infusion) }\end{array}$ \\
\hline
\end{tabular}

\section{b Phase 3 Study Design}

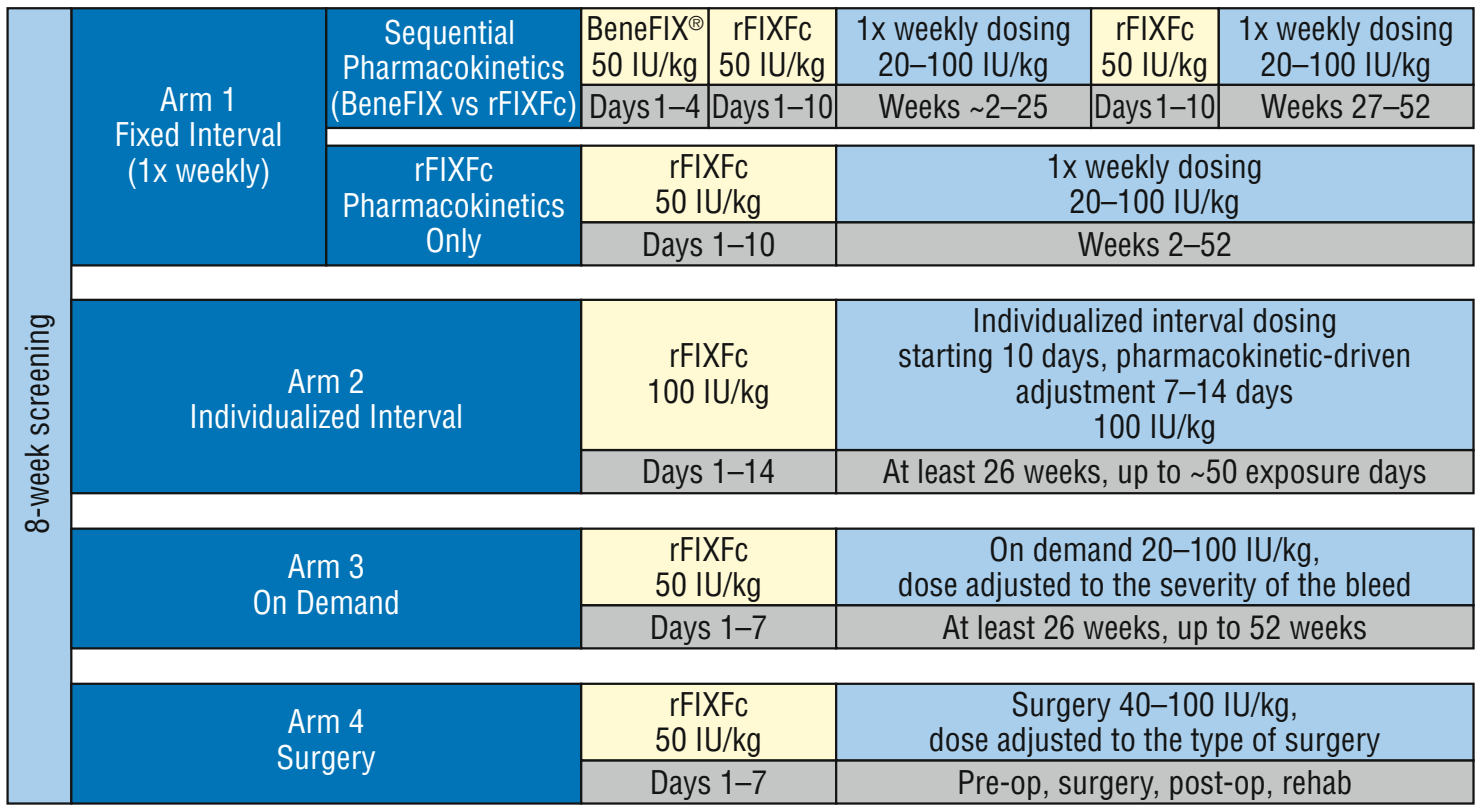

\section{c. rFIXFc Pharmacokinetic Sampling Schemes}

\begin{tabular}{|c|c|}
\hline Study arm/subgroup & Sampling timepoints \\
\hline Phase 1/2a 12.5-100 IU/kg rFIXFc & $\begin{array}{l}\text { Pre-dose; end of infusion ( } 10 \mathrm{~min}), 15 \mathrm{~min} \text { after the end of infusion, } \\
1,3,6,24,48,72,96,120,168 \text { and } 240 \mathrm{~h}(288 \mathrm{~h} \text { and } 336 \mathrm{~h} \text { if FIX } \\
\text { activity was above baseline at day } 13)\end{array}$ \\
\hline Phase 3 Arm 1/sequential pharmacokinetics ${ }^{a}$ & Pre-dose; $10 \mathrm{~min}, 1,3,6,24,48,96,144,168,192$ and $240 \mathrm{~h}^{\mathrm{c}}$ \\
\hline Phase 3 Arm 1/non-sequential pharmacokinetics ${ }^{a}$ & Pre-dose; $10 \mathrm{~min}, 3,24,48,96,168$ and $240 \mathrm{~h}$ \\
\hline Phase 3 Arm $2^{b}$ & Pre-dose; $10 \mathrm{~min}, 3,24,48,96,168,240,288$ and $336 \mathrm{~h}$ \\
\hline Phase 3 Arms 3 and $4^{a}$ & Pre-dose; $10 \mathrm{~min}, 3,24,48,96$ and $168 \mathrm{~h}$ \\
\hline
\end{tabular}

Fig. 1 Study design for a phase 1/2a and $\mathbf{b}$ phase 3 clinical trials and $\mathbf{c}$ recombinant factor IX Fc (rFIXFc) sampling schemes

dose records. For patients whose lowest observed FIX activity was $<1 \%$ (LLOQ), the baseline FIX activity was set at 0 ; for patients whose lowest observed FIX activity was between 1-2 \%, the baseline FIX activity was set at the lowest observed FIX activity. The study enrolment was limited to subjects with baseline FIX activity $\leq 2 \%$.
For each individual subject, the observed FIX activity was subtracted from baseline activity and the decayed residual activity, if any, to obtain the corrected FIX activity. Residual activity was defined as pre-dose activity minus baseline FIX activity. For subjects in the Arm 1 sequential pharmacokinetic subgroup who underwent 
pharmacokinetic assessment with the comparator FIX product (BeneFIX ${ }^{\circledR}$; Pfizer Inc, New York, NY, USA) prior to $\mathrm{rFIXFc}$ pharmacokinetic assessment, the residual activity was decayed using the individual subject's BeneFIX terminal first-order decay rate estimated by the non-compartmental analysis in Phoenix ${ }^{\mathrm{TM}}$ WinNonlin 6.2 (Pharsight, Sunnyvale, CA, USA). For any subjects who did not have a BeneFIX pharmacokinetic assessment, the residual activity was decayed using the average BeneFIX terminal first-order decay rate estimated from the Arm 1 sequential pharmacokinetic subgroup.

\subsection{Modelling Strategy and Datasets}

Demographic and clinical factors collected and examined in the analysis included age, body weight (BW), race, height, human immunodeficiency virus (HIV) and hepatitis $\mathrm{C}$ virus (HCV) status, IgG1 and albumin concentrations, haematocrit (HCT) level, FIX genotype and blood type. A summary of categorical factors and baselines for continuous factors is listed in Table 1.

The pharmacokinetic dataset was split into the modelling dataset, which was used to build the population pharmacokinetic model, and the validation dataset, which was used to qualify the final model. The modelling dataset for rFIXFc included 1,400 FIX activity records from 135 baseline pharmacokinetic profiles in both phase $1 / 2 \mathrm{a}$ and 3 studies, as well as 21 repeat pharmacokinetic profiles that were collected at week 26 from the Arm 1 sequential pharmacokinetic subgroup in the phase 3 study. The validation dataset included 1,027 trough/peak FIX activity records from the phase 3 study, excluding the records during and after surgeries. Peak/trough collection times were recorded by patients retrospectively in their electronic diary following the clinic visit. A summary of the modelling and validation datasets is listed in Table 2.

The modelling strategy was a two-step approach. The first step was to build the population pharmacokinetic model using the modelling dataset and the second step was to validate the model with goodness-of-fit plots, bootstrapping, a visual prediction check (VPC) and the trough/ peak validation dataset [16]. As a comparison, the rFIXFc model using the full dataset, which combined the modelling and validation dataset, was also developed.

\subsection{Population Pharmacokinetic Modelling}

NONMEM $^{\circledR} 7$ version 1.0 (ICON Development Solutions, Ellicott City, MD, USA) with an Intel Fortran compiler (version 12) was used for the population pharmacokinetic model development. Statistical program R (version 2.15.0; R Foundation for Statistical Computing, Vienna, Austria) was used to compile NONMEM datasets and generate
Table 1 Summary of categorical demographic and clinical factors and baseline values for continuous demographic and clinical factors

\begin{tabular}{|c|c|}
\hline Parameter & Value \\
\hline \multicolumn{2}{|c|}{ Categorical demographic and clinical factors } \\
\hline \multicolumn{2}{|l|}{$\operatorname{Race}[n(\%)]$} \\
\hline American Indian or Alaska Native & $1(0.74)$ \\
\hline Asian & $30(22.2)$ \\
\hline Black or African American & $12(8.9)$ \\
\hline White & $82(60.7)$ \\
\hline Other & $10(7.4)$ \\
\hline \multicolumn{2}{|l|}{$\mathrm{HIV}[n(\%)]$} \\
\hline Yes & $5(3.7)$ \\
\hline No & $130(96.3)$ \\
\hline \multicolumn{2}{|l|}{$\operatorname{HCV}[n(\%)]$} \\
\hline Yes & $52(38.5)$ \\
\hline No & $83(61.5)$ \\
\hline \multicolumn{2}{|l|}{ Blood type $[n(\%)]$} \\
\hline A & $72(53.3)$ \\
\hline $\mathrm{B}$ & $21(15.6)$ \\
\hline $\mathrm{AB}$ & $7(5.2)$ \\
\hline $\mathrm{O}$ & $35(25.9)$ \\
\hline \multicolumn{2}{|l|}{ Genotype $[n(\%)]$} \\
\hline Missense & $75(55.5)$ \\
\hline Nonsense & $24(17.8)$ \\
\hline Frameshift & $18(13.3)$ \\
\hline Splice mutation & $4(3.0)$ \\
\hline Others & $14(10.4)$ \\
\hline
\end{tabular}

Continuous demographic and clinical factors (baseline)

Age (years)

$\begin{array}{ll}n & 135 \\ \text { Mean (SD) } & 34.6(15.2) \\ \text { Median (range) } & 31.3(12.1-76.8) \\ \text { Weight (kg) } & 135 \\ n & 75.9(20.1) \\ \text { Mean (SD) } & 73.3(45-186.7) \\ \text { Median (range) } & \\ \text { IgG1 (mg/mL) } & 123 \\ n & 7.68(2.62) \\ \text { Mean (SD) } & 7.19(3.34-18.3) \\ \text { Median (range) } & \\ \text { Albumin (g/L) } & 134 \\ n & 46(3.43) \\ \text { Mean (SD) } & 46(30-56) \\ \text { Median (range) } & \\ \text { HCT (volume/volume) } & 135 \\ n & 0.44(0.05) \\ \text { Mean (SD) } & 0.44(0.21-0.55) \\ \text { Median (range) } & \end{array}$

HCT haematocrit, $H C V$ hepatitis $\mathrm{C}$ virus, HIV human immunodeficiency virus, $I g G 1$ immunoglobulin $\mathrm{G} 1, n$ number of subjects, $S D$ standard deviation 
Table 2 Summary of modelling and validation datasets

\begin{tabular}{lllll}
\hline Dataset & No. of patients & No. of FIX activity records & Age (years) [median (range)] & Body weight (kg) [median (range)] \\
\hline Modelling dataset & $\begin{array}{l}12 \text { (phase 1/2a) } \\
123 \text { (phase 3) }\end{array}$ & 1,400 & $31.3(12.1-76.8)$ & $73.3(45.0-186.7)$ \\
Validation dataset & 100 (phase 3) $^{\mathrm{a}}$ & 1,027 & $30.7(12.1-71.6)$ & $72.5(45.2-186.7)$ \\
\hline
\end{tabular}

FIX factor IX

${ }^{a}$ Phase $1 / 2$ a was a single-dose study; no peaks/troughs were collected

graphics. Perl Speaks NONMEM (PsN, version 3.5.3) [17] was used to conduct bootstrapping. PsN and Xpose 4 [18] were used to perform VPC.

A first-order conditional estimation with interaction method (FOCEI) was used to estimate population pharmacokinetic parameters. Inter-individual variability (IIV) was modelled using the exponential function. The inclusion of IIV terms on pharmacokinetic parameters was tested sequentially, with the most significant objective function value $(\mathrm{OFV})$ reduction $(P<0.005)$ entering the model first. Inter-occasion variability (IOV) [19] was also evaluated. For the modelling dataset, two occasions were assigned to the baseline pharmacokinetic profiling at week 1 and repeat pharmacokinetic profiling at week 26, respectively. For the full dataset, six occasions were defined according to the data density. Residual errors were modelled as combined proportional and additive errors.

Plots of IIV versus covariates were used to screen for potential demographic and clinical factors that affect rFIXFc pharmacokinetics. For continuous covariates, scatter plots of ETA (IIV code used in NONMEM) versus covariates were overlaid with a non-parametric locally weighted smoother Loess line to determine functional relationships; for categorical covariates, box-and-whisker plots were used to identify potential differences between groups (data not shown). A clear trend of positive or negative slopes and noteworthy correlation coefficients (data not shown) would suggest a possible influence by the continuous covariates; pronounced differences among the groups would suggest a possible influence by the categorical covariates. After identifying potential covariates, a full stepwise forward addition $(P<0.005)$ and backward elimination $(P<0.001)$ procedure was conducted for covariate modelling.

Besides statistical considerations, model selection was also aided by goodness-of-fit plots, including DV versus population prediction (PRED), DV versus individual prediction (IPRED), conditional weighted residual (CWRES) versus TIME and PRED plots [20, 21]. Other diagnostics also helped to select the proper model, including parameter precision, ETA and CWRES distribution and shrinkage $[22,23]$.

\subsection{Model Qualification}

Bootstrapping was conducted with 1,000 datasets generated by random sampling through replacement [24]. Nonparametric medians and $95 \%$ (2.5th and 97.5th percentile) confidence intervals (CIs) of pharmacokinetic parameters were obtained and compared with final model estimates.

To check the predictive performance of the model, VPC was performed to obtain 1,000 simulated pharmacokinetic profiles [24]. Medians and 10th and 90th percentiles of simulated and observed FIX activities, stratified by dose (50 and $100 \mathrm{IU} / \mathrm{kg}$ ), were plotted.

The trough/peak validation dataset was used to check the predictability of the model $[16,24,25]$. Specifically, the model was used to derive Bayesian feedback predictions of FIX activities at trough/peak time points by setting MAXEVAL $=0$ in the NONMEM control stream. The mean relative prediction error (an indicator of accuracy) was calculated using Eq. 3.

Relative prediction error $=\frac{1}{N} \sum_{i=1}^{i=N} \frac{\mathrm{DV}-\mathrm{IPRED}}{\mathrm{DV}}$

\section{Results}

\subsection{Structural Model and Evaluation of IIV}

Based on previous conventional pharmacokinetic analyses of rFIXFc [6], a two-compartment model appropriately described individual pharmacokinetics, hence a two-compartment model was evaluated first followed by a threecompartment model. IIV (ETA, $\eta$ values) was assumed for clearance (CL) and volume of compartment $1\left(V_{1}\right)$. A covariance between $\mathrm{CL}$ and $V_{1}$ was also included. The three-compartment model resulted in a reduction of OFV by over 400 units (for additional four parameters) compared with the two-compartment model, and thus was selected as the base model (Fig. 2). Primary pharmacokinetic parameters included CL, $V_{1}$, volumes of compartment $2\left(V_{2}\right)$ and $3\left(V_{3}\right)$, and inter-compartmental clearance between compartments 1 and $2\left(Q_{2}\right)$, as well as between 1 and $3\left(Q_{3}\right)$. The inclusion of IIV for the rest of the 


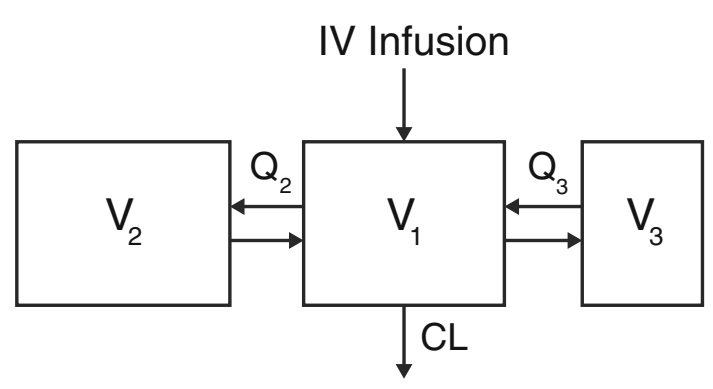

Fig. 2 Three-compartment pharmacokinetic model. $C L$ clearance, $I V$ intravenous, $Q_{2}$ inter-compartmental clearance between compartments 1 and 2, $Q_{3}$ inter-compartmental clearance between compartments 1 and $3, V_{1}$ volume of compartment $1, V_{2}$ volume of compartment $2, V_{3}$ volume of compartment 3

pharmacokinetic parameters $\left(V_{2}, V_{3}, Q_{2}\right.$ and $\left.Q_{3}\right)$ led to further improvement in the model fitting. However, IIV on $Q_{3}$ was associated with a high standard error $(87 \%)$, indicating that the data could not support a precise estimation of IIV on $Q_{3}$, which was thus not included in the model. No additional covariance between IIV of pharmacokinetic parameters could be estimated with precision, thus the only covariance between IIV retained in the model was the covariance between IIV on CL and $V_{1}$.

\subsection{Evaluation of IOV}

Since the Arm 1 sequential pharmacokinetic subgroup had repeat pharmacokinetic profiles at week 26 in addition to baseline pharmacokinetic profiles at week 1 , IOV was evaluated with baseline pharmacokinetics as occasion 1 and repeat pharmacokinetics as occasion 2. The inclusion of IOV on CL significantly improved the model with a reduction of OFV by 171.6 units. The inclusion of IOV on both CL and $V_{1}$ achieved an additional OFV drop of 41.6 units, whereas IOV on $V_{2}$ or $Q_{2}$ did not improve the model fit $(P>0.05)$. The IOV on $\mathrm{V}_{3}$ improved the model fit at $P<0.005$ but with a large percentage of relative standard error (78.4\%); therefore, IOV was only included for CL and $V_{1}$.

Pairwise comparisons of $\mathrm{CL}$ and $V_{1}$ estimates for baseline and repeat pharmacokinetics, derived from the base model with IOV, were plotted in Fig. 3. The changes of either CL or $V_{1}$ between the two occasions were random and small with only one exception, and the mean CL or $V_{1}$ for the two occasions were similar.

Overall, the inclusion of IOV reduced the corresponding IIV on CL and $V_{1}$ from 24.0 and $29.6 \%$ to 21.1 and $24.2 \%$, respectively. The inclusion of IOV also reduced proportional and additive residual errors from $12.1 \%$ and $0.30 \mathrm{IU} / \mathrm{dL}$ to $10.5 \%$ and $0.24 \mathrm{IU} / \mathrm{dL}$, respectively. The base model with IOV provided a reasonable fit to the data, and explained the small, as well as random, pharmacokinetic changes between occasions studied in the trial, and therefore was chosen for further covariate modelling.

\subsection{Covariate Modelling}

Based on ETA versus covariate plots, BW, albumin and race on CL, and 'study' on $V_{2}$ were speculated to be potential covariates. Covariate modelling included BW on all pharmacokinetic parameters, albumin on $\mathrm{CL}$, and 'study' on $V_{2}$ and CL. BW was assessed for all pharmacokinetic parameters because it is an important physiology factor. 'Study' was assessed on CL because of the importance of CL.

A full stepwise forward addition and backward elimination procedure was performed. Following the forward covariate inclusion, the full covariate model was identified with BW on CL and $V_{1}$, and 'study' on $V_{2}$. However, 'study' on $V_{2}$ was removed following the backward elimination procedure $(P>0.001)$.

Further, the potential residual variability difference between the phase $1 / 2 \mathrm{a}$ and 3 studies was tested by including two sets of proportional and additive errors for two studies in the residual error model. No significant reduction in OFV was observed (13.7 units, $d f=2$ ). Therefore, although the phase $1 / 2 \mathrm{a}$ and phase 3 studies have different dosing and sampling schemes, the population pharmacokinetic modelling did not suggest a pharmacokinetic difference between the two studies.

\subsection{Final Model}

The final model of rFIXFc had IIV on CL $/ V_{1} / Q_{2} / V_{2} / V_{3}$ but not $Q_{3}$, IOV on CL and $V_{1}$ and $\mathrm{BW}$ as a covariate on $\mathrm{CL}$ and $V_{1}$. The model described the data well (Fig. 4). There were no outstanding trends observed in the CWRES plots and most CWRES were randomly distributed between -2 and 2, indicating overall small discrepancies between measured FIX activities and population predictions (Fig. 4c, d). Population pharmacokinetic parameter estimates, IIV and IOV, as well as residual errors, were estimated with precision, evidenced by narrow $95 \%$ CIs for each pharmacokinetic parameter (Table 3). The IIVs for CL and $V_{1}$ were 17.7 and $21.7 \%$, respectively, which are low to moderate, and the IOVs for CL and $V_{1}$ were low at 15.1 and $17.4 \%$, respectively.

The magnitude of ETA shrinkage on the IIVs was moderate $(<30 \%$ for all pharmacokinetic parameters with IIV terms), while the magnitude of ETA shrinkage on the IOVs was occasion-specific, moderate at first occasion (around $30 \%$ on $\mathrm{CL}$ and $V_{1}$ ) and higher at occasion 2 (around $70 \%$ ) because there were fewer pharmacokinetic 
Fig. 3 Pairwise comparison of baseline and repeat pharmacokinetics: a clearance (CL) and $\mathbf{b}$ volume of compartment $1\left(V_{1}\right)$ estimates occasion variability. Red line represents the mean with the base model with inter-
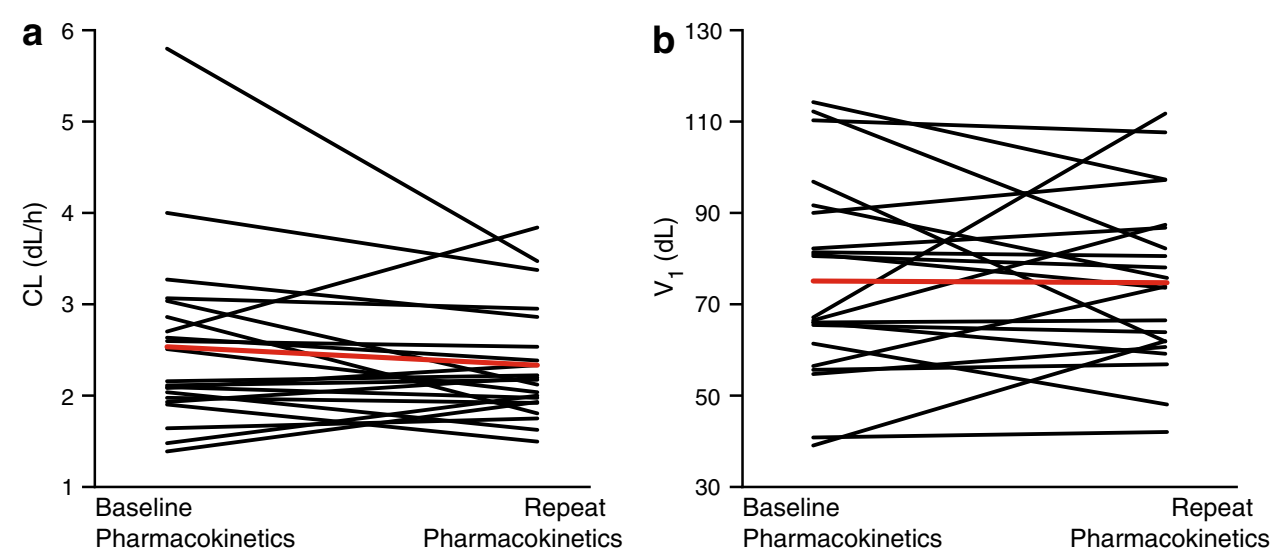

Fig. 4 Goodness-of-fit plots of the final model. Black solid line is the unity line in $\mathbf{a}$ and $\mathbf{b}$. Red solid line represents the linear regression line in $\mathbf{a}$ and $\mathbf{b}$ and the Loess smoother in $\mathbf{c}$ and $\mathbf{d}$; dependent variable (DV) is corrected factor IX (FIX) activity and unit is IU/dL; PRED is the population FIX activity prediction and unit is IU/dL; IPRED is the individual FIX activity prediction and unit is IU/dL; CWRES is conditional weighted residual; Time is the time after dose and unit is hour
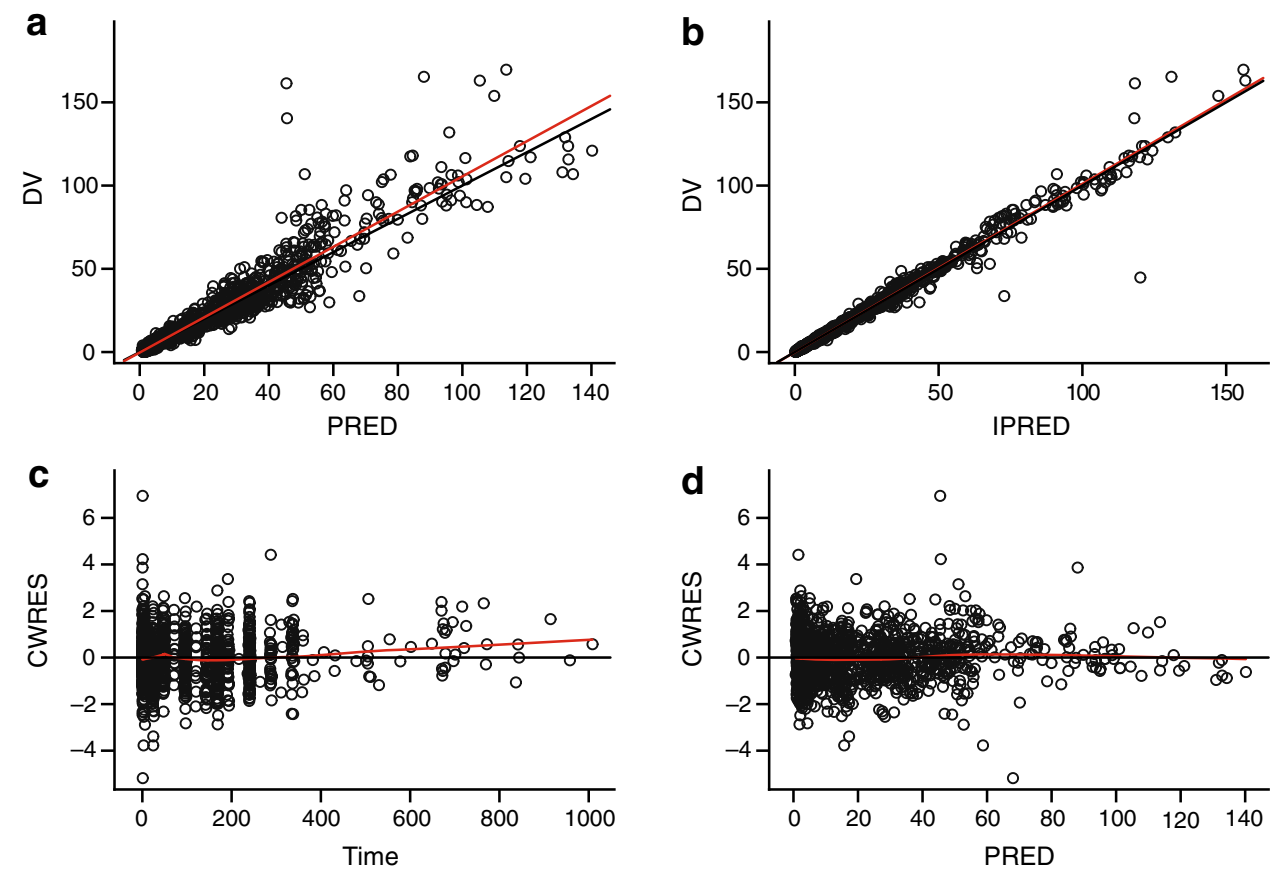

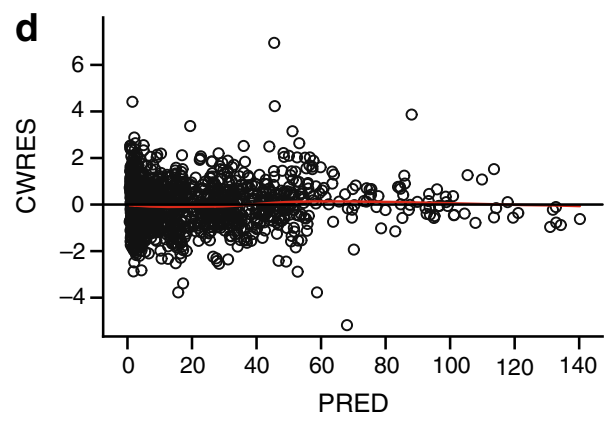

profiles for the second occasion (21 for occasion 2 repeat pharmacokinetics vs 135 for occasion 1 baseline pharmacokinetics). The distributions of ETAs and CWRES showed approximate normal distribution centred around zero without apparent skewness (data not shown). This was consistent with the ETABAR $P$ values, all of which were non-significant $(P>0.05)$.

\subsection{Model Qualification}

Non-parametric bootstrapping was applied to the final model to assess the model stability. Bootstrapping generated medians and CIs for the pharmacokinetic parameters, IIV and IOV estimates (Table 3). The median values from the bootstrapping were very similar to the model estimates for all the pharmacokinetic parameters.
The graphic results of the VPC of the final model stratified by the dose are presented in Fig. 5. The median and $80 \%$ interval (10th to 90th percentile) time-activity observed and predicted profiles nearly overlapped, indicating that the final model was able to reproduce both the central tendency and variability of the observed FIX activity time profiles.

The predictive capability of the final model was further evaluated using a validation dataset, which contains the trough/peak FIX activity records that were not included in the modelling dataset. The final model was used to derive the individual predictions for the trough and peak observations. Individual predictions showed good correlation $\left(R^{2}=0.9857, P<0.001\right)$ with the observations (Fig. 6). The mean relative prediction error was low at $-3.23 \%$, indicating that the final model was qualified to predict 
Table 3 Summary of recombinant factor IX Fc (rFIXFc) population pharmacokinetic final model

$B W$ body weight, $C I$ confidence interval, $C L$ clearance, $I I V$ interindividual variability, $I O V$ interoccasion variability, $Q_{2}$ intercompartmental clearance of compartment $2, Q_{3}$ intercompartmental clearance of compartment $3, V_{l}$ volume of compartment $1, V_{2}$ volume of compartment $2, V_{3}$ volume of compartment 3

a $95 \% \mathrm{CI}$, non-parametric $95 \% \mathrm{CI}$ from bootstrap results with 1,000 datasets

${ }^{\mathrm{b}}$ IIV calculated as

$\sqrt{\text { variance }} \times 100$

c IOV calculated as

$\sqrt{\text { variance }} \times 100$

\begin{tabular}{lll}
\hline Parameter & Model estimate & Bootstrap median $\left(95 \% \mathrm{CI}^{\mathrm{a}}\right)$ \\
\hline $\mathrm{CL}=$ typical $\mathrm{CL} \times\left(\frac{\mathrm{BW}}{73}\right)^{0.436}$ & & \\
Typical CL for a $73 \mathrm{~kg}$ patient $(\mathrm{dL} / \mathrm{h})$ & 2.39 & $2.39(2.29,2.49)$ \\
BW exponent on CL & 0.436 & $0.437(0.272,0.584)$ \\
$V_{1}=$ typical $V_{1} \times\left(\frac{\mathrm{BW}}{73}\right)^{0.396}$ & & \\
Typical $V_{1}$ for a $73 \mathrm{~kg}$ patient $(\mathrm{dL})$ & 71.4 & $71.2(58.5,76.0)$ \\
BW exponent on $V_{1}$ & 0.396 & $0.390(0.169,0.580)$ \\
$Q_{2}(\mathrm{dL} / \mathrm{h})$ & 1.67 & $1.66(1.35,1.89)$ \\
$V_{2}(\mathrm{dL})$ & 87.0 & $87.0(79.0,95.5)$ \\
$Q_{3}(\mathrm{dL} / \mathrm{h})$ & 39.3 & $39.0(16.6,141)$ \\
$V_{3}(\mathrm{dL})$ & 39.9 & $41.2(36.6,52.4)$ \\
IIV on CL, \% & 17.7 & $17.5(11.8,22.4)$ \\
IOV on CL, \% & 15.1 & $15.0(10.7,19.1)$ \\
IIV on $V_{1}, \%$ & 21.7 & $22.4(15.5,32.1)$ \\
IOV on $V_{1}, \%$ & 17.4 & $16.5(8.7,22.8)$ \\
IIV on $Q_{2}, \%$ & 35.8 & $35.0(22.6,45.8)$ \\
IIV on $V_{2}, \%$ & 46.2 & $45.9(38.0,55.3)$ \\
IIV on $V_{3}, \%$ & 37.7 & $37.9(30.2,54.3)$ \\
Correlation between IIV on CL and $V_{1}, \%$ & 75.6 & 74.8 \\
Proportional residual error, \% & 10.6 & $10.4(8.64,12.0)$ \\
Additive residual error, IU/dL & 0.24 & $0.24(0.17,0.31)$ \\
\hline
\end{tabular}

rFIXFc pharmacokinetics in the haemophilia B patient population.

\subsection{Full Dataset Model}

Further, a population pharmacokinetic model of rFIXFc was also built based on the full dataset, including both pharmacokinetic profile and trough/peak data. The population parameter estimates of the resulting model, as well as IIV and IOV (Online Resource Table S1), were comparable with those of the final model derived from the modelling dataset (Table 3). The goodness-of-fit plots indicated that the model also described the data adequately (Online Resource Fig. S1). A slightly greater over-prediction of FIX activity in the lower range $(<10 \mathrm{IU} / \mathrm{dL})$ was observed for the VPC of the full dataset model (Fig. 5c, d).

\section{Discussion}

This is the first systematic population pharmacokinetic modelling of rFIXFc in patients with haemophilia B. A three-compartment model described the pharmacokinetics of rFIXFc well. For a typical $73 \mathrm{~kg}$ patient, $V_{1}$ for rFIXFc at $71.4 \mathrm{dL}$ is larger than the plasma volume, which is around $30 \mathrm{dL}$ for a typical adult, indicating that rFIXFc is not limited in the plasma for the initial distribution phase after intravenous administration, similar to that of FIX, which is known to bind to collagen IV in the subendothelium [26]. The IIVs for CL and $V_{1}$ were low to moderate at 17.7 and $21.7 \%$, respectively, which are consistent with those reported for plasma-derived FIX (23\% for CL and $19 \%$ for $V_{1}$ ) [12]. Residual errors were small with a proportional error of $10.6 \%$ and additive error of $0.24 \mathrm{IU} / \mathrm{dL}$. The proportional residual error is similar to the inter-assay variability of the one-stage aPTT clotting assay. The small IIV and residual errors indicate that the model described the data adequately and rFIXFc pharmacokinetics do not vary substantially among patients. The estimated IOVs for CL and $V_{1}$ were 15.1 and $17.4 \%$, respectively, similar to those reported for plasma-derived FIX (15\% for CL and $12 \%$ for $V_{1}$ ) [12]. The small and randomly distributed IOVs on CL and $V_{1}$ indicate that rFIXFc pharmacokinetics are relatively stable at different occasions.

The approach of using the model to estimate baseline and differentiate baseline from pre-dose residual activity for each individual was investigated. However, population modelling cannot reliably separate baseline from residual activity because not every FIX activity profile returned to baseline at the last sampling time point [i.e. the baseline (endogenous) and exogenous signals were confounded]. We also investigated setting baseline activity at $0,0.5$ or an individualized baseline. The individualized baseline resulted in relatively conservative pharmacokinetic estimates and more accurate prediction of the trough levels in individual subjects. Therefore, an individualized baseline was 
Fig. 5 Visual predictive check for $\mathbf{a}$ and $\mathbf{b}$ the final model derived from the modelling dataset, and $\mathbf{c}$ and $\mathbf{d}$ the model derived from the full dataset. Black solid lines and red dashed lines are 10th, 50th and 90th percentiles of the observation (black) and simulation (red), respectively; a and $\mathbf{c}$ represent dose groups of $50 \mathrm{IU} / \mathrm{kg}$; $\mathbf{b}$ and d represent dose groups of $100 \mathrm{IU} / \mathrm{kg}$. FIX factor IX
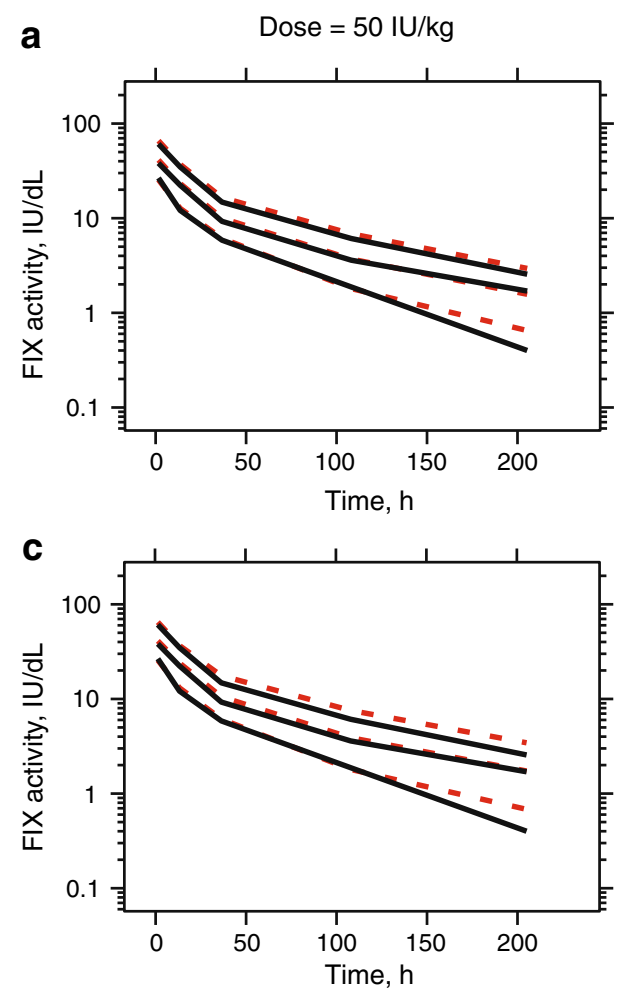
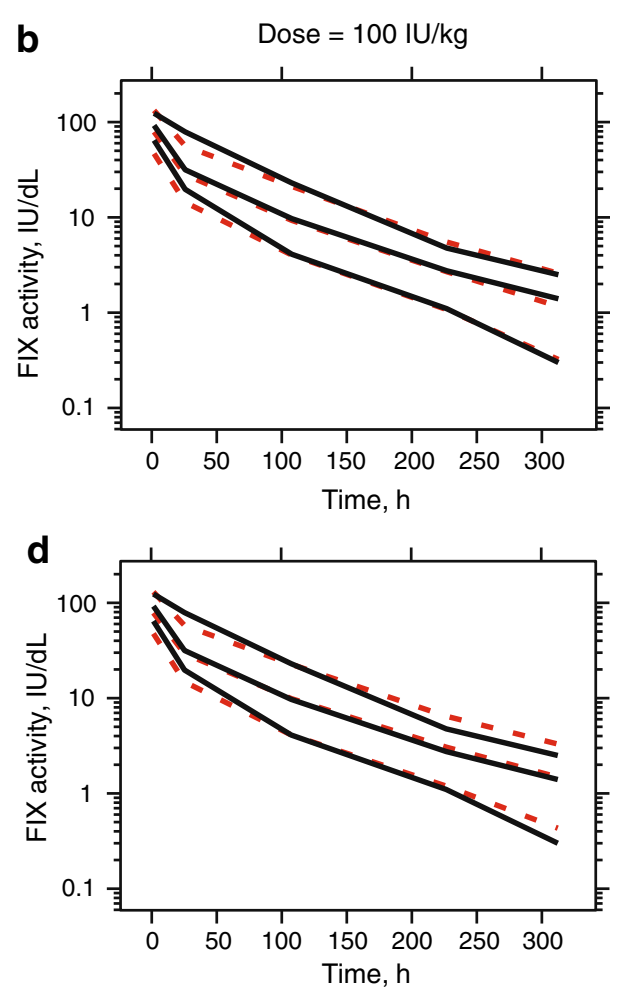

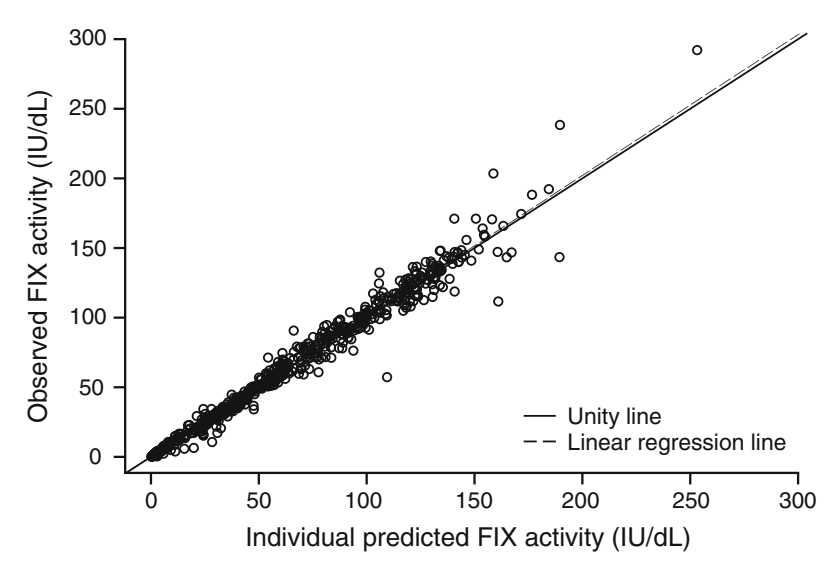

Fig. 6 Prediction of trough/peak factor IX (FIX) activities in the validation dataset

chosen to handle the activity data in the population pharmacokinetic modelling, which was also utilized in the conventional pharmacokinetic analysis [8].

BW on CL and $V_{1}$ was the only covariate that showed a statistically significant impact on rFIXFc pharmacokinetics. It was suggested that the exponent of a physiological or pharmacokinetic parameter should not revolve around a fixed number [27]. Hence, the exponents of BW on CL and $V_{1}$ were estimated during the modelling instead of being fixed at presumed values, e.g. 0.75 for $\mathrm{CL}$ and 1 for $V_{1}$. The estimated BW exponents for CL and $V_{1}$ in the final model were markedly lower at 0.436 and 0.396 , respectively. Furthermore, inclusion of BW as a covariate decreased IIV for CL by only $3.4 \%$ and IIV for $V_{1}$ by only $2.5 \%$, suggesting that a considerable portion of the variability was not explained by BW.

The limited impact of BW was not unique to rFIXFc pharmacokinetics, which was also observed for BeneFIX in the phase 3 study (data not shown). The weak correlation between $\mathrm{BW}$ and pharmacokinetics in our studies differs from a previous report, which showed that BW, with an exponent of 0.7 on CL, accounted for a significant portion of the variability in BeneFIX pharmacokinetics in a two-compartment population pharmacokinetic model [28]. The discrepancy probably can be explained by the different populations studied, i.e. adult patients $(>19$ years) in our study versus pooled data from 111 children ( $\leq 15$ years), including 53 infants $(<2$ years), and 80 adults ( $>15$ years). This previous report represents a wider range for age and BW than in our study. A recently published paper reported that BeneFIX pharmacokinetics in 56 patients aged 4-56 years and weighing $18-133 \mathrm{~kg}$, described also by a three-compartment model, had allometric exponent of CL terms of 0.66 and volume terms of 0.64 [29]. The slightly reduced allometric exponent of CL compared with the previous report [28] might also be explained by the difference of age and BW range studied.

Data splitting is a useful internal model validation approach in population pharmacokinetic modelling [24]. Because intensive pharmacokinetic profile data are used to predict subsequent trough/peak sparse data in the clinic, the 
data in this study were split into a modelling dataset including the intensive pharmacokinetic profile data from all subjects at week 1 and week 26 and a validation dataset including the sparse peak and trough data throughout the phase 3 study. To verify that our modelling strategy was robust, i.e. building the model with the baseline/repeat pharmacokinetic profiles without additional trough/peak FIX activity records, we also built the model using the full dataset consisting of all the FIX activity records from both the modelling and validation datasets. The two models were highly comparable with $<10 \%$ difference in the pharmacokinetic parameters, IIV and IOV estimates (Table 3 and Online Resource Table S1). The comparability between the two models was also demonstrated by the similar VPC plots for the two models (Fig. 5). FIX activities in the lower range $(<10 \mathrm{IU} / \mathrm{dL})$ were slightly more over-predicted by the full dataset model. This difference might be attributed to the imprecise recordings of the peak/trough collection time in the full dataset, which was retrospectively recorded by patients in their electronic diary following the clinic visit. The final model derived from the modelling dataset is slightly more accurate in predicting trough levels, which is essential for maintenance of the therapeutic efficacy. Therefore, the final model derived from the modelling dataset is robust and predictive to be used for simulation of the dosing regimens for rFIXFc.

Finally, the population pharmacokinetic predictions were largely consistent with the results derived from the conventional two-stage pharmacokinetic analysis, which used a two-compartment model, although a minority $(\sim 14 \%)$ of the pharmacokinetic profiles could also be described by a three-compartment model. The ambiguity in the model selection in the conventional pharmacokinetic analysis was at least partially due to the different sampling schemes in different study arms. Such ambiguity was avoided using population pharmacokinetic modelling. The post hoc estimates from this population pharmacokinetic analysis were very similar to the results from the conventional pharmacokinetic analysis (Online Resource Table $\mathrm{S} 2$; [8]). For example, the geometric mean $t_{1 / 2}$ values estimated in population pharmacokinetics and conventional pharmacokinetics are 81.1 and $82.1 \mathrm{~h}$, respectively. The highly comparable pharmacokinetic parameters derived from a two-compartment conventional pharmacokinetic analysis and a three-compartment population pharmacokinetic analysis suggest that the contribution of the third compartment to rFIXFc pharmacokinetics was probably limited but nevertheless provided better profile definition for the more complex population modelling. The advantage of developing a population pharmacokinetic model for rFIXFc is that because FIX activity is considered as a surrogate for efficacy [12], the model can be utilized for dosing regimen simulation taking into account IIV and IOV. Further, the population pharmacokinetic model combined with individual sparse pharmacokinetic data can be used to derive an individualized dosing regimen through Bayesian estimation, which can alleviate the requirement for extensive sampling. Since haemophilia is a lifelong disease impacting children as well as adults, the benefit of pharmacokinetics-tailored dosing regimens based on data from limited blood sampling is of great interest to the haemophilia community.

\section{Conclusion}

This is the first population pharmacokinetic analysis that systematically characterized the pharmacokinetics of longlasting rFIXFc in patients with haemophilia B. The disposition of rFIXFc was well described by a three-compartment model with low to moderate IIV and IOV. Body weight was found to be the only statistically significant but weak covariate on $\mathrm{CL}$ and $V_{1}$ with limited impact. The qualified population pharmacokinetic model for rFIXFc is appropriate and predictive, providing a valuable tool to evaluate and optimize dosing regimens of rFIXFc for the treatment of patients with haemophilia B.

Acknowledgments We would like to acknowledge James Woodworth, Mark Rogge and Srividya Neelakantan for helpful discussion and advice; Mark McLaughlin and John Hotaling for dataset preparation; Snejana Krassova, Geoffrey Allen and Alvin Luk for trial management; and the physicians and patients for their participation in the trials. LD, SL, IN and HJ are employees of and hold equity interest in Biogen Idec. TL and JG are paid consultants of Biogen Idec. Editorial assistance was provided by Katie Gersh, $\mathrm{PhD}$, of MedErgy and was funded by Biogen Idec.

Open Access This article is distributed under the terms of the Creative Commons Attribution Noncommercial License which permits any noncommercial use, distribution, and reproduction in any medium, provided the original author(s) and the source are credited.

\section{References}

1. Mannucci PM, Tuddenham EG. The hemophilias-from royal genes to gene therapy. N Engl J Med. 2001;344(23):1773-9.

2. Giangrande P. Haemophilia B: Christmas disease. Expert Opin Pharmacother. 2005;6(9):1517-24.

3. Srivastava A, Brewer AK, Mauser-Bunschoten EP, Key NS, Kitchen S, Llinas A, et al. Guidelines for the management of hemophilia. Haemophilia. 2013;19(1):e1-47.

4. Roopenian DC, Akilesh S. FcRn: the neonatal Fc receptor comes of age. Nat Rev Immunol. 2007;7(9):715-25.

5. Kuo TT, Aveson VG. Neonatal Fc receptor and IgG-based therapeutics. MAbs. 2011;3(5):422-30.

6. Shapiro AD, Ragni MV, Valentino LA, Key NS, Josephson NC, Powell JS, et al. Recombinant factor IX-Fc fusion protein $(\mathrm{rFIXFc})$ demonstrates safety and prolonged activity in a phase 1/2a study in hemophilia B patients. Blood. 2012;119(3):666-72. 
7. Peters RT, Low SC, Kamphaus GD, Dumont JA, Amari JV, Lu $\mathrm{Q}$, et al. Prolonged activity of factor IX as a monomeric Fc fusion protein. Blood. 2010;115(10):2057-64.

8. Powell JS, Pasi J, Ragni MV, Ozelo MC, Valentino LA, Mahlangu JN, et al. Study of recombinant factor IX Fc fusion protein in hemophilia B. N Engl J Med. 2013;369:2313-23.

9. Beal SL. Ways to fit a PK model with some data below the quantification limit. J Pharmacokinet Pharmacodyn. 2001;28(5): 481-504.

10. Byon W, Smith MK, Chan P, Tortorici MA, Riley S, Dai H, et al. Establishing best practices and guidance in population modeling: an experience with an internal population pharmacokinetic analysis guidance. CPT Pharmacometrics Syst Pharmacol. 2013;2:e51.

11. Bergstrand M, Karlsson MO. Handling data below the limit of quantification in mixed effect models. AAPS J. 2009;11(2): 371-80.

12. Bjorkman S, Ahlen V. Population pharmacokinetics of plasmaderived factor IX in adult patients with haemophilia B: implications for dosing in prophylaxis. Eur $\mathrm{J}$ Clin Pharmacol. 2012;68(6):969-77.

13. Bjorkman S, Carlsson M, Berntorp E. Pharmacokinetics of factor IX in patients with haemophilia B. Methodological aspects and physiological interpretation. Eur J Clin Pharmacol. 1994;46(4): 325-32.

14. Bjorkman S, Shapiro AD, Berntorp E. Pharmacokinetics of recombinant factor IX in relation to age of the patient: implications for dosing in prophylaxis. Haemophilia. 2001;7(2):133-9.

15. Carlsson M, Bjorkman S, Berntorp E. Multidose pharmacokinetics of factor IX: implications for dosing in prophylaxis. Haemophilia. 1998;4(2):83-8.

16. Guidance for industry on population pharmacokinetics; availability. Food and Drug Administration, HHS. Notice. Fed Regist. 1999;64(27):6663-4.

17. Lindbom L, Ribbing J, Jonsson EN. Perl-speaks-NONMEM (PsN) - a Perl module for NONMEM related programming. Comput Methods Programs Biomed. 2004;75(2):85-94.

18. Jonsson EN, Karlsson MO. Xpose-an S-PLUS based population pharmacokinetic/pharmacodynamic model building aid for NONMEM. Comput Methods Programs Biomed. 1999;58(1): $51-64$.
19. Karlsson MO, Sheiner LB. The importance of modeling interoccasion variability in population pharmacokinetic analyses. J Pharmacokinet Biopharm. 1993;21(6):735-50.

20. Wade JR, Edholm M, Salmonson T. A guide for reporting the results of population pharmacokinetic analyses: a Swedish perspective. AAPS J. 2005;7(2):45.

21. Ette EI, Ludden TM. Population pharmacokinetic modeling: the importance of informative graphics. Pharm Res. 1995;12(12): 1845-55.

22. Savic RM, Karlsson MO. Importance of shrinkage in empirical Bayes estimates for diagnostics: problems and solutions. AAPS J. 2009;11(3):558-69.

23. Xu XS, Yuan M, Karlsson MO, Dunne A, Nandy P, Vermeulen A. Shrinkage in nonlinear mixed-effects population models: quantification, influencing factors, and impact. AAPS J. 2012;14(4):927-36.

24. Sherwin CM, Kiang TK, Spigarelli MG, Ensom MH. Fundamentals of population pharmacokinetic modelling: validation methods. Clin Pharmacokinet. 2012;51(9):573-90.

25. Brendel K, Dartois C, Comets E, Lemenuel-Diot A, Laveille C, Tranchand B, et al. Are population pharmacokinetic and/or pharmacodynamic models adequately evaluated? A survey of the literature from 2002 to 2004. Clin Pharmacokinet. 2007;46(3): 221-34.

26. Gui T, Lin HF, Jin DY, Hoffman M, Straight DL, Roberts HR, et al. Circulating and binding characteristics of wild-type factor IX and certain Gla domain mutants in vivo. Blood. 2002;100(1): 153-8.

27. Mahmood I. Theoretical versus empirical allometry: facts behind theories and application to pharmacokinetics. J Pharm Sci. 2010;99(7):2927-33.

28. Udata C, Sullivan S, Kelly P, Roth DA, Meng X. Population pharmacokinetic modeling of BeneFIX in pediatric and adult patients with hemophilia B demonstrates weight as an important factor contributing to inter-patient PK variability. Blood. 2008;112(11):443-4.

29. Bjorkman S. Population pharmacokinetics of recombinant factor IX: implications for dose tailoring. Haemophilia. 2013;19(5): $753-7$. 